\title{
Over-the-scope clip used to control bleeding from a duodenal ulcer
}

The over-the-scope clip (OTSC) is a new device that was initially developed for the closure of perforations of the gastrointestinal tract, but has since been used for a variety of indications including closure of fistulas $[1,2]$. We report the use of an OTSC to control persistent bleeding from a duodenal ulcer.

A 57-year-old woman was transferred to our hospital in February 2011 because of acute renal failure with hyperglycemic acidosis secondary to type I diabetes. During her admission she developed a non-ST elevation myocardial infarction (NSTEMI) and was commenced on double platelet aggregation inhibitor therapy with acetylsalicylic acid and clopidogrel.

She was readmitted to our hospital 3 weeks later with acute gastrointestinal bleeding, presenting as melena and hematemesis. Immediate endoscopy showed a duodenal ulcer (Forrest Ia) with arterial bleeding $(\bullet$ Fig. 1) that was stopped with injection of saline and adrenaline $(1: 10000)$ and subsequent application of hemoclips (Olympus, Hamburg, Germany). A Helicobacter pylori quick test was positive and the patient was treated with intravenous pantoprazole twice daily and commenced on eradication therapy. The patient was stable overnight on the intensive care unit and underwent a further endoscopy the next day. During this examination, rebleeding was encountered and was treated with epinephrine, further hemoclips, and injection of fibrin ( $\bullet$ Fig. 2 ).

The patient chose not to undergo the surgical intervention that was proposed to her at this stage, instead opting strongly for treatment using an OTSC (Ovesco, Tübingen, Germany). This was attempted the following day, by which time the hemoclips had again been lost and an actively bleeding ulcer (now Forrest Ib) was present ( Fig.3). Because it was not possible to get the whole ulcer into the OTSC overcap ( Fig.4a), the OTSC was centered on the vessel, which was laterally placed ( Fig.4b). Following application of the OTSC the bleeding stopped immediately. Follow-up endoscopies 3 days and 6 days later showed no further signs

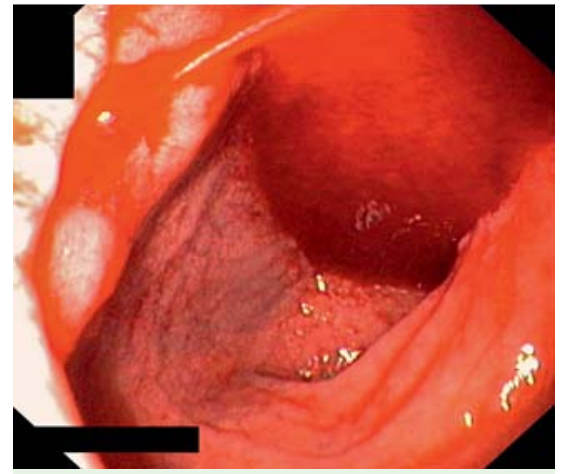

Fig. 1 The Forrest la duodenal ulcer at presentation showing evidence of arterial bleeding.

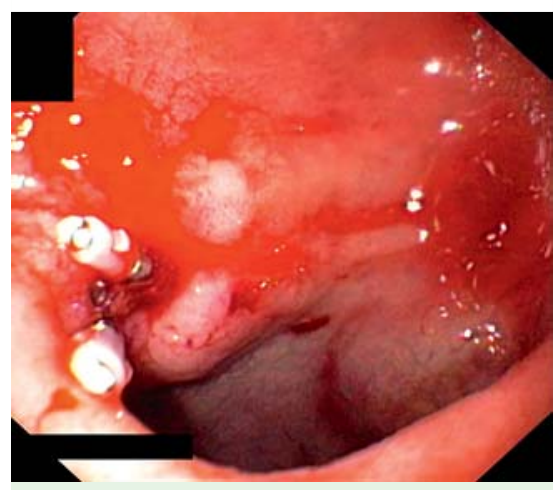

Fig. 2 The ulcer on day 2 following treatment with epinephrine, fibrin injection, and further placement of hemoclips.

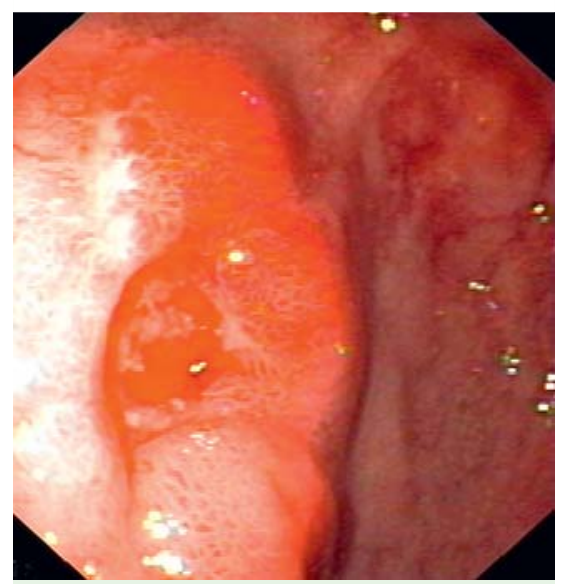

Fig. 3 The ulcer on day 3, by which time it had become Forrest Ib and the hemoclips had again been lost.
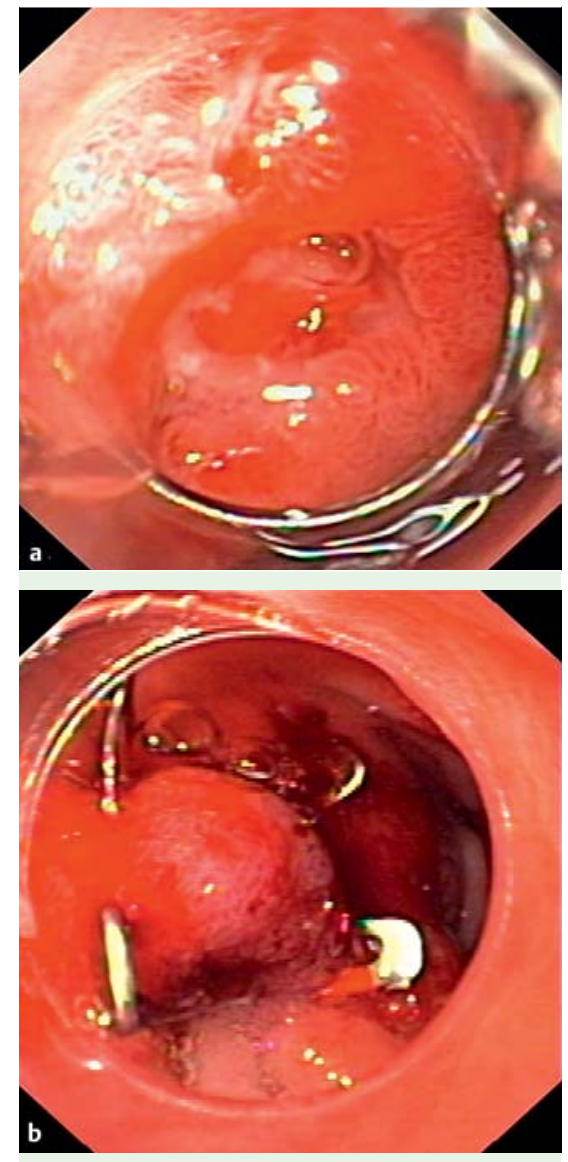

Fig.4 An attempt was made to place an overthe-scope clip (OTSC), but because a the ulcer was too large to fit completely into the OTSC overcap; $\mathbf{b}$ the OTSC was centered on the laterally placed vessel.

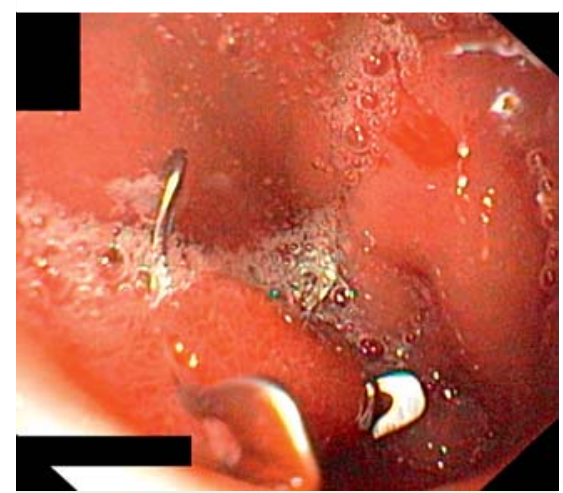

Fig. 5 Follow-up endoscopy performed at 3 months showed that the over-the-scope clip (OTSC) was still in situ. 
of bleeding. The patient was discharged and returned for follow-up endoscopies 4 weeks and 12 weeks later, at which times the OTSC was found to be still in situ ( Fig. 5).

Endoscopy_UCTN_Code_TTT_1AO_2AD

\section{Competing interests: None}

M. Peters, P. Meyer, J. Martin, A. Poerschke, S. Rupp, K.-H. Kirschstein

Jewish Hospital, Department

of Medicine II - Gastroenterology

and Diabetology, Berlin, Germany

\section{References}

1 Kirschniak A, Kratt T, Stüker D et al. A new endoscopic over-the-scope clip system for treatment of lesions and bleeding in the GI tract: first clinical experiences. Gastrointest Endosc 2007; 66: $162-167$

2 Repici A, Arezzo A, De Caro G et al. Clinical experience with a new endoscopic over-thescope clip system for use in the GI tract. Dig Liver Dis 2009; 41: 406-410
Bibliography

Dol http://dx.doi.org/

10.1055/s-0031-1291515

Endoscopy 2012; 44: E36-E37

(C) Georg Thieme Verlag KG

Stuttgart · New York

ISSN 0013-726X

\section{Corresponding author}

\section{Peters, MD}

Chefarzt der Klinik für Innere Medizin II Gastroenterologie und Diabetologie Jüdisches Krankenhaus Berlin 13347 Berlin

Fax: +49-30-49942512

michael.peters@jkb-online.de 